\title{
Is the effect of tinnitus on auditory steady-state response amplitude mediated by attention?
}

\author{
Eugen Diesch ${ }^{1 *}$, Martin Andermann ${ }^{2}$ and Andre Rupp ${ }^{2}$ \\ ${ }^{1}$ Department of Clinical and Cognitive Neuroscience, Central Institute of Mental Health, Medical Faculty Mannheim, Heidelberg University, Mannheim, Germany \\ 2 Department of Neurology, Section of Biomagnetism, University Hospital Heidelberg, Heidelberg, Germany
}

\section{Edited by: \\ Jos J. Eggermont, University of \\ Calgary, Canada}

Reviewed by:

Larry Roberts, McMaster University,

Canada

Grant Searchfield, The University of Auckland, New Zealand

${ }^{*}$ Correspondence:

Eugen Diesch, Department of

Clinical and Cognitive Neuroscience

Central Institute of Mental Health,

Medical Faculty Mannheim,

Heidelberg University, Square J5,

D-68159 Mannheim, Germany.

e-mail: eugen.diesch@

zi-mannheim.de
Objectives: Auditory steady-state response (ASSR) amplitude enhancement effects have been reported in tinnitus patients. As ASSR amplitude is also enhanced by attention, the effect of tinnitus on ASSR amplitude could be interpreted as an effect of attention mediated by tinnitus. As N1 attention effects are significantly larger than those on the ASSR, if the effect of tinnitus on ASSR amplitude were due to attention, there should be similar amplitude enhancement effects in tinnitus for the N1 component of the auditory-evoked response. Methods: MEG recordings which were previously examined for the ASSR (Diesch et al., 2010a) were analyzed with respect to the N1m component. Like the ASSR previously, the N1m was analyzed in the source domain (source space projection). Stimuli were amplitude-modulated (AM) tones with one of three carrier frequencies matching the tinnitus frequency or a surrogate frequency $1 \frac{112}{2}$ octave above the audiometric edge frequency in controls, the audiometric edge frequency, and a frequency below the audiometric edge. Single AM-tones were presented in a single condition and superpositions of three AM-tones differing in carrier and modulation frequency in a composite condition. Results: In the earlier ASSR study (Diesch et al., 2010a), the ASSR amplitude in tinnitus patients, but not in controls, was significantly larger in the (surrogate) tinnitus condition than in the edge condition. Patients showed less evidence than controls of reciprocal inhibition of component ASSR responses in the composite condition. In the present study, N1m amplitudes elicited by stimuli located at the audiometric edge and at the (surrogate) tinnitus frequency were smaller than $\mathrm{N} 1 \mathrm{~m}$ amplitudes elicited by sub-edge tones both in patients and controls. The relationship of the $\mathrm{N} 1 \mathrm{~m}$ response in the composite condition to the $\mathrm{N} 1 \mathrm{~m}$ response in the single condition indicated that reciprocal inhibition among component $\mathrm{N} 1 \mathrm{~m}$ responses was reduced in patients compared against controls. Conclusions: In the present study, no evidence was found for an N1-amplitude enhancement effect in tinnitus. Compared to controls, reciprocal inhibition is reduced in tinnitus patients. Thus, as there is no effect on $\mathrm{N} 1 \mathrm{~m}$ that could potentially be attributed to attention, it seems unlikely that the enhancement effect of tinnitus on ASSR amplitude could be accounted for in terms of attention induced by tinnitus.

Keywords: tinnitus, auditory cortex, hyperexcitability, attention

\section{INTRODUCTION}

In previous studies it has been found that the amplitude of the magnetically recorded auditory steady-state response (ASSR) is enhanced for frequencies in the tinnitus frequency region (Diesch et al., 2004, 2010a,b). This enhancement effect may extend to nearby frequencies below this region (Diesch et al., 2004; Wienbruch et al., 2006). The ASSR may be elicited by trains of clicks and tone pips or by beats and sinusoidally amplitudemodulated (AM) tones. The source of the ASSR has been localized to the primary auditory cortex in the medial partition of Heschl's gyrus (Mäkelä and Hari, 1987; Gutschalk et al., 1999; Ross et al., 2000, 2002; Schoonhoven et al., 2003). Amplitude enhancement in tinnitus of stimulus-driven activity in the afferent auditory pathway may be interpreted to reflect the workings of gain control mechanisms inherent in the functioning of the subcortical auditory nuclei and the auditory cortex (Salvi et al., 2000; Syka, 2002; Eggermont and Roberts, 2004; Parra and Pearlmutter, 2007; Sun et al., 2009; Norena, 2010). However, because ASSR amplitude is also enhanced by attention (Ross et al., 2004; BidetCaulet et al., 2007; Gander et al., 2007, 2010; Müller et al., 2009), the effect of tinnitus on ASSR amplitude could in principle be an effect of attention in disguise. Those affected by tinnitus may direct attention to the auditory modality, to the ear affected by tinnitus, or even to the frequency or frequency range optimally corresponding to the equivalent tinnitus frequency or the dominant frequencies of the tinnitus spectrum. The two hypotheses are similar in that amplitude enhancement by attention of stimulus-evoked neural activity in the auditory pathway may be conceptualized in terms of attention-driven gain adjustment (Hillyard et al., 1998; Winkowski and Knudsen, 
2006; Fritz et al., 2007; Winkowski and Knudsen, 2008). They differ in that the gain control hypothesis proper posits a modular autoregulatory process within the afferent auditory pathway while the gain-adjustment-by-attention hypothesis postulates that sensory activity is amplified by an interactive top-down process.

Not only the ASSR, but also several components of the transient auditory-evoked response, among them the auditory N1, are modulated by attention (Näätänen, 1992). The N1 component of the auditory-evoked potential and its magnetic counterpart, the N1m, occurs with a latency of approximately $100 \mathrm{~ms}$ in response to stimulus onset, stimulus offset, and stimulus change (Näätänen and Picton, 1987). The N1(m) receives contributions from the primary auditory cortex (Steinschneider et al., 1994), but the gravitational center of the $\mathrm{N} 1(\mathrm{~m})$ source has been localized to an auditory belt area in the planum temporale posterior to Heschl's gyrus (Pantev et al., 1995; Lütkenhöner and Steinsträter, 1998; Godey et al., 2001). While not much is known about attention effects on the offset and the change $\mathrm{N} 1(\mathrm{~m})$, there is ample evidence that the onset $\mathrm{N} 1(\mathrm{~m})$ shows attention enhancement effects (electrical: Hillyard et al., 1973; Picton and Hillyard, 1974), magnetic: (Arthur et al., 1991; Rif et al., 1991; Woldorff et al., 1993; Fujiwara et al., 1998). Moreover, N1 attention enhancement effects are significantly larger than ASSR attention enhancement effects (Okamoto et al., 2011). Thus, if the effect of tinnitus on ASSR amplitude were actually due to attention, similar, if not larger, amplitude enhancement effects should be found for the $\mathrm{N} 1(\mathrm{~m})$ component of the auditory-evoked response.

Previous studies on the N1 in tinnitus that reported tinnitusrelated amplitude decrease rather than amplitude increase (e.g., Jacobson et al., 1991; Colding-Jorgensen et al., 1992; Jacobson and McCaslin, 2003; Walpurger et al., 2003) do not necessarily provide evidence against the attention hypothesis, as $1000 \mathrm{~Hz}$ sine tones exclusively were used as stimuli, whereas for most individuals affected the dominant tinnitus frequency is above $1000 \mathrm{~Hz}$. Norena et al. (1999) also presented $1000 \mathrm{~Hz}$ tones and reported an increase of the slope of the $\mathrm{N} 1$ amplitude-level function in tinnitus patients and Kadner et al. (2002) obtained a steeper N1 amplitude-level function in tinnitus patients for tonal stimuli at the tinnitus frequency than for stimuli at lower frequencies. Thus, there remains a possibility that the N1 amplitude show attentionrelated enhancement effects mediated by tinnitus, especially with tonal stimuli close to the tinnitus frequency.

Here, we examine the $\mathrm{N} 1 \mathrm{~m}$ responses in a data-set that we have studied previously for the ASSR. Specifically, we look at $\mathrm{N} 1 \mathrm{~m}$ amplitude enhancement effects in tinnitus. If these were absent, it would seem unlikely that the tinnitus-associated ASSR amplitude enhancement effects observed previously (Diesch et al., 2004, 2010a,b) are mediated by attention ${ }^{1}$. Secondly, we compare the amplitude of the N1m elicited by composite stimuli

\footnotetext{
${ }^{1}$ Following the logic laid out by Okamoto et al. (2011), the sustained field could also have been examined. We were prevented from doing this because an analog highpass filter with a cutoff frequency of $0.03 \mathrm{~Hz}$ was used in the original recording. Extraction and analysis of the sustained field require DC recordings.
}

to the linear superposition sum of the amplitudes of the N1m responses elicited by the individual component stimuli. The frequency bands of the auditory system are not independent, but rather constitute a multi-stage lateral, or reciprocal, inhibiton network (Shamma and Symmes, 1985; Müller and Scheich, 1988; Vater et al., 1992; Rhode and Greenberg, 1994; Suga, 1995; Sutter et al., 1999; Jen et al., 2002; Kopp-Scheinpflug et al., 2002). In the auditory cortex, integration of thalamocortical and intracortical inputs occurs over a range that spans a large proportion of the audible spectrum (Schulze and Langner, 1999; Kaur et al., 2004; Metherate et al., 2005). Attention affects sensory processing not only by increasing the gain, but also by increasing the selectivity of the receptive fields of single units (Fritz et al., 2007) and neural populations (Okamoto et al., 2007; Kauramäki et al., 2007; Neelon et al., 2011). Top-down control of reciprocal inhibition may be the mechanism or one of the mechanisms by means of which this increase of selectivity is accomplished. If it were, a putative tinnitus-related effect of attention on N1m amplitude should reduce the ratio of $\mathrm{N} 1 \mathrm{~m}$ response amplitude to composite to the superposition sum of N1m response amplitudes to component stimuli.

\section{MATERIALS AND METHODS}

MEG recordings of auditory-evoked responses that were previously examined for the ASSR (Diesch et al., 2010a) were analyzed with regard to the N1m component. Patients were included in the study if their tinnitus had lasted for six months or longer and featured a dominant tonal or quasi-tonal component that an equivalent tinnitus frequency of at least $1000 \mathrm{~Hz}$ could be determined for. Controls did not present with tinnitus. Both patients and controls were selected only if they showed some high-frequency hearing loss above an audiometric edge, where the audiometric edge was defined as that point on the frequency axis at which the hearing loss function exhibited its largest downward slope. Thirty-six subjects, 18 tinnitus patients and 18 healthy controls, participated in the original study. Subjects gave written informed consent following procedures approved by the ethics committee of the University of Heidelberg. Because musicians exhibit larger auditory-evoked response amplitudes and, therefore, deliver better signal-to-noise ratios than non-musicians when presented with spectrally complex sounds (Pantev et al., 1998a; Kuriki et al., 2006), half of the participants, eight of the patients and 10 of the controls, were selected to be musicians. Participants were assigned to the musician group if either they worked as professional musicians or earned a score of 25 or higher on the Advanced Measures of Music Audiation (AMMA) test (Gordon, 1989, 1998), or both. Thus, there were four groups, musicians with tinnitus (group MT, $n=8$, mean age $=44.4$ years, $\mathrm{SD}=12.7$, range: $21-63$ ), non-musicians with tinnitus (group $\mathrm{NT}, n=10$, mean age $=51.4$ years, $\mathrm{SD}=11.0$, range: $21-58)$, musicians without tinnitus (group $\mathrm{MN}, N=10$, mean age $=$ 36.1 years, $\mathrm{SD}=11.3$, range: $21-52)$, and non-musicians without tinnitus (group NN, $n=8$, mean age $=44.3$ years, $\mathrm{SD}=$ 13.7, range: $23-58$ ). The groups did not differ significantly in sex and handedness, but tinnitus patients were marginally older than healthy controls $\left[F_{(3,32)}=2.68, p<0.07\right]$. Patients and controls did not significantly differ in musicality. As a result of the 
selection procedure, musicians had higher AMMA scores than non-musicians $\left[F_{(1,28)}=64.5, p<0.0001\right]$.

The absolute threshold of hearing was obtained for 12 tonal frequencies between $125 \mathrm{~Hz}$ and $15 \mathrm{kHz}$ (Figure 1). Summary indices of hearing loss both in the stimulated and the nonstimulated ear were determined by computing averages across frequencies, i.e., from $125 \mathrm{~Hz}$ to $746 \mathrm{~Hz}$ for low frequency and from $1183 \mathrm{~Hz}$ to $15 \mathrm{kHz}$ for high frequency hearing loss. The equivalent tonal frequency of the tinnitus was determined using a recursive two-interval forced-choice procedure, with pure tones presented in both of the intervals, which has proven a reliable measure of tinnitus frequency (Henry and Meikle, 2000; Henry et al., 2000). The tinnitus minimum masking level (TMML) was determined as the difference between the level that was just sufficient for masking the tinnitus and the threshold level at which the masker was just audible by using a narrow-band ( 0.7 critical bands) "low-noise noise" (Kohlrausch et al., 1997; Dau et al., 1999) masker the center frequency of which was equated with the tinnitus frequency. The German version of the Tinnitus Questionnaire (Hallam et al., 1988; Hallam, 1996) published by Goebel and Hiller (1994, 1998) with its subscales of tinnitus intrusiveness, cognitive and emotional distress, auditory and perceptual diffculties, somatic complaints, and sleep disturbances was used to measure tinnitus severity.

Hearing loss (Figure 1) was more pronounced for patients than controls both for low $\left[125 \ldots 746 \mathrm{~Hz}: F_{(1,32)}=6.7\right.$, $p<0.02]$ and for high frequencies $\left[1.18 \ldots 15 \mathrm{kHz}: F_{(1,32)}=\right.$ $10.6, p<0.005]$. For high frequencies, the stimulated ear was significantly worse than the non-stimulated ear $\left[F_{(1,32)}=8.28\right.$, $p<0.01]$. Musicality and its interactions were not significant. Audiometric edge frequency did not differ significantly between patients and controls or musicians and non-musicians. Musicians with tinnituss did not differ from non-musicians with tinnitus with regard to tinnitus frequency, TMML, the tinnitus questionnaire total score, and the tinnitus questionnaire subscale scores.

The stimuli for the MEG-study were AM sine tones with a duration of $8192 \mathrm{~ms}, 20 \mathrm{~ms}$ onset and offset cosine ramps, and a modulation depth of $100 \%$. The offset-to-onset inter-stimulus interval was allowed to vary randomly between 800 and $1200 \mathrm{~ms}$. There were three individually adjusted carrier frequencies matching the tinnitus frequency in patients and the "surrogate tinnitus frequency" $1 \frac{1}{2}$ octaves above the audiometric edge frequency in controls (tinnitus condition: T), the audiometric edge frequency (edge condition: E), and a frequency $1 \frac{1 / 2}{2}$ octaves below
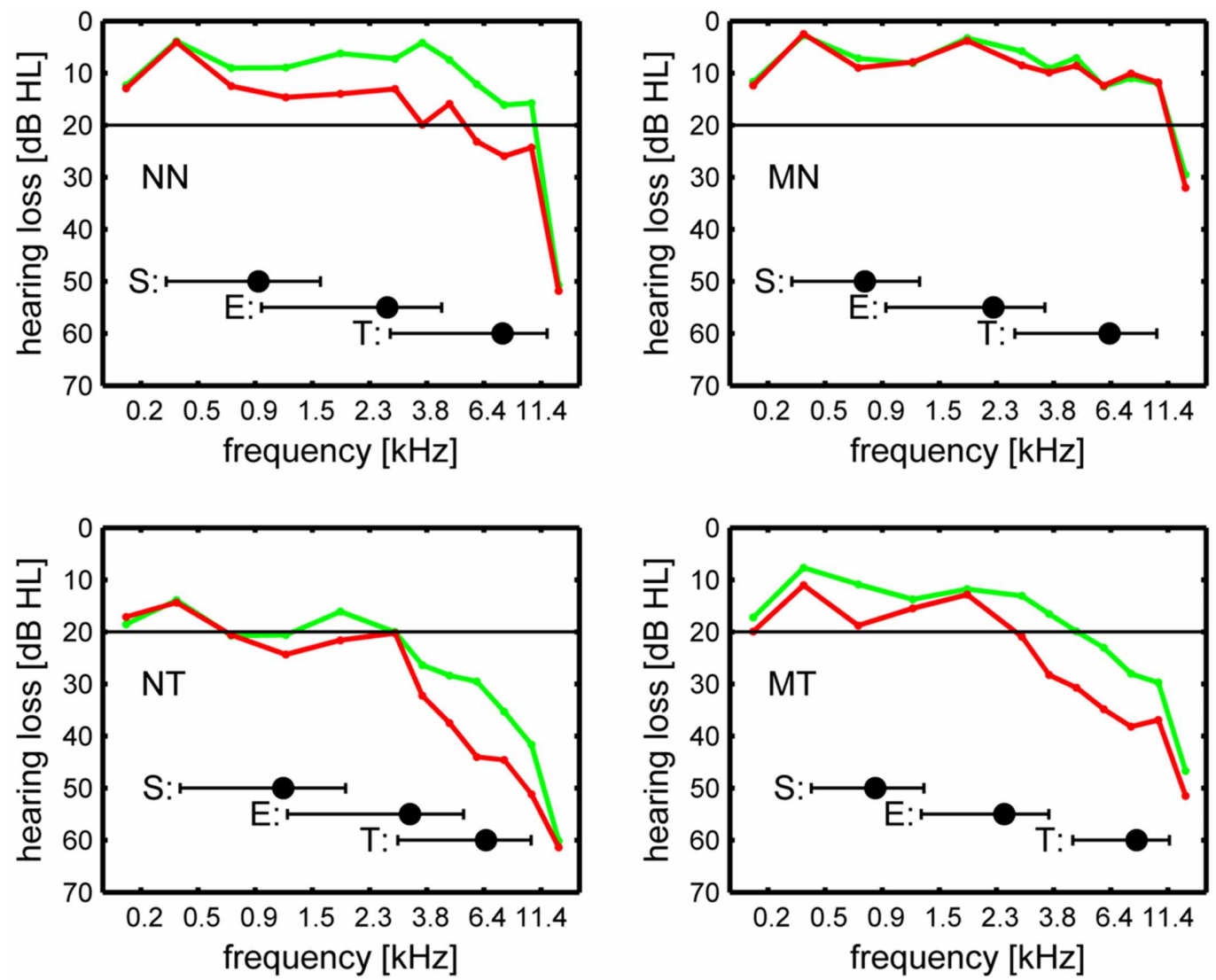

FIGURE 1 | Hearing loss, in dB HL, in the stimulated ear (red line) and the non-stimulated ear (green line). The inset bars show mean and

the sub-edge condition (S), the edge condition (E), and the (surrogate) tinnitus condition (T). NN: non-musicians without tinnitus, MN: musicians without tinnitus, NT: non-musicians with tinnitus, MT: musicians with tinnitus. 
the audiometric edge (sub-edge condition: $S$ ). There were three modulation frequencies set to $38.6,40.6$, and $42.6 \mathrm{~Hz}$ (conditions 38,40 , and 42). All possible combinations of carrier and modulation frequencies occurred equally often both in a single tone (S38, S40, S42, E38, E40, E42, T38, T40, T42) and a composite tone condition (S38_E40_T42, S40_E42_T38, S42_E38_T40). Composite tones were created through linear superposition of single tones.

With regard to composite tones, there is an important difference between the ASSR and the N1m. Single tones elicited an ASSR with a frequency equal to the stimulus modulation rate. As to be expected (Lins and Picton, 1995; Lins et al., 1996; John et al., 1998; Fujiki et al., 2002; Kaneko et al., 2003), composite tones elicited three simultaneous SSRs with frequencies equal to the three stimulus modulation rates that were extracted separately using appropriate bandpass filters (Diesch et al., 2010a). Both single and composite tones elicited an N1m. However, it is not possible to decompose the composite tones N1m into component responses that one could attribute to the component tones. As a consequence, putative reciprocal inhibition among component $\mathrm{N} 1 \mathrm{~m}$ responses cannot be measured in the way that reciprocal inhibition between multiple ASSR components can be measured. For the ASSR, the amplitudes of the component responses in the composite stimulus condition may be compared to the respective same-modulation rate response in the single stimulus condition, with amplitude reduction in the composite stimulus condition indicating reciprocal inhibition and amplitude enhancement indicating reciprocal facilitation (Diesch et al., 2010a). For the N1m, one has to find an estimate of the composite stimulus condition $\mathrm{N} 1 \mathrm{~m}$ amplitude free of putative reciprocal inhibition or facilitation effects. An estimate may be obtained by computing the source amplitude of the equivalent current source fitted to the linear superposition of the field distributions accounted for by the single stimulus condition N1m sources which, in turn, may be approximated by simply computing the sum of the single stimulus condition N1m source amplitudes (Diesch and Luce, 1997).

The neuromagnetic field was recorded with a 122-channel gradiometer (Neuromag, Helsinki, Finland). The analog signal was lowpass-filtered $(330 \mathrm{~Hz})$, highpass-filtered $(0.03 \mathrm{~Hz})$, and digitized with a digitization rate of $1000 \mathrm{~Hz}$. The stimuli were delivered monaurally through a plastic tube to the ear with the larger amount of hearing loss above $1 \mathrm{kHz}$. The stimulus presentation sequence was randomized. The stimulus level was set to $50 \mathrm{~dB}$ sensation level ( $\mathrm{dB} \mathrm{SL}$ ), measured with the participant seated under the dewar, both for single tone stimuli and composite tone component stimuli. The stimulus level was lowered, if the resulting level of the composite stimuli was uncomfortable to the participant or the extent of the hearing loss was such that the limits of the transmission system were reached. The minimum stimulus level used was $35 \mathrm{~dB}$ SL. The stimulus level was lowered for four participants of group NT $(3 \times 35,40 \mathrm{~dB} S \mathrm{SL})$, three participants of group MT $(2 \times 35,40 \mathrm{~dB} \mathrm{SL})$, and one participant of group NN ( $40 \mathrm{~dB} \mathrm{SL}$ ). However, for any one participant the same stimulus level (re sensation level) was used throughout. Due to their more pronounced high frequency hearing loss, the need to lower the stimulus level arose more frequently for tinnitus patients than for controls. Stimuli were equated re sensation level rather than in perceived loudness, because both ASSR and N1m amplitudes were intended to be studied as indicators of auditory system gain.

In accordance with the earlier ASSR analysis (Diesch et al., 2010a), the N1m was analyzed in the source domain using source space projection. To obtain the requisite spatial projection filter, the raw MEG signal was highpass-filtered at $1 \mathrm{~Hz}$ and lowpass-filtered at $30 \mathrm{~Hz}$. Epochs of $500 \mathrm{~ms}$ duration including a $200 \mathrm{~ms}$ prestimulus baseline interval were extracted from the continuous data. Epochs exceeding $3000 \mathrm{fT} / \mathrm{cm}$ in amplitude were discarded. A grand average was computed across all stimulus conditions. Dipole source analysis was conducted using the BESA Ver. 5.2 software (MEGIS Software GmbH, Munich, Germany). A source model with two equivalent current dipoles, one in either hemisphere, was selected. Source fits were deemed acceptable if the equivalent sources were located in the supratemporal plane and the residual variance was $15 \%$ or less. The average residual variance obtained was $8.9 \%(\mathrm{SD}=3.73)$. Because of a bad source fit resulting from low signal amplitude one of the NT group participants was excluded from the N1m analysis. Following Hämälainen et al. (1993) and Robinson (1989), the unfiltered raw data were projected into the source domain, with the dipoles of the source model being used as the spatial projection filter. This resulted in two continuous source domain data streams representing activity of the auditory cortices of the left and the right hemisphere at the locations of the $\mathrm{N} 1 \mathrm{~m}$ sources. Figure 2 illustrates the outcome of the application of the source space projection filter, not to the raw data, however, but to the grand average that was computed across all stimulus conditions.

The source domain time series were exported to MATLAB for further analysis. The exported continuous source domain data were highpass-filtered $(1 \mathrm{~Hz})$, lowpass-filtered $(30 \mathrm{~Hz})$, and selectively averaged with hemisphere and stimulus type, i.e., the three carrier frequencies of the single stimulus condition and the

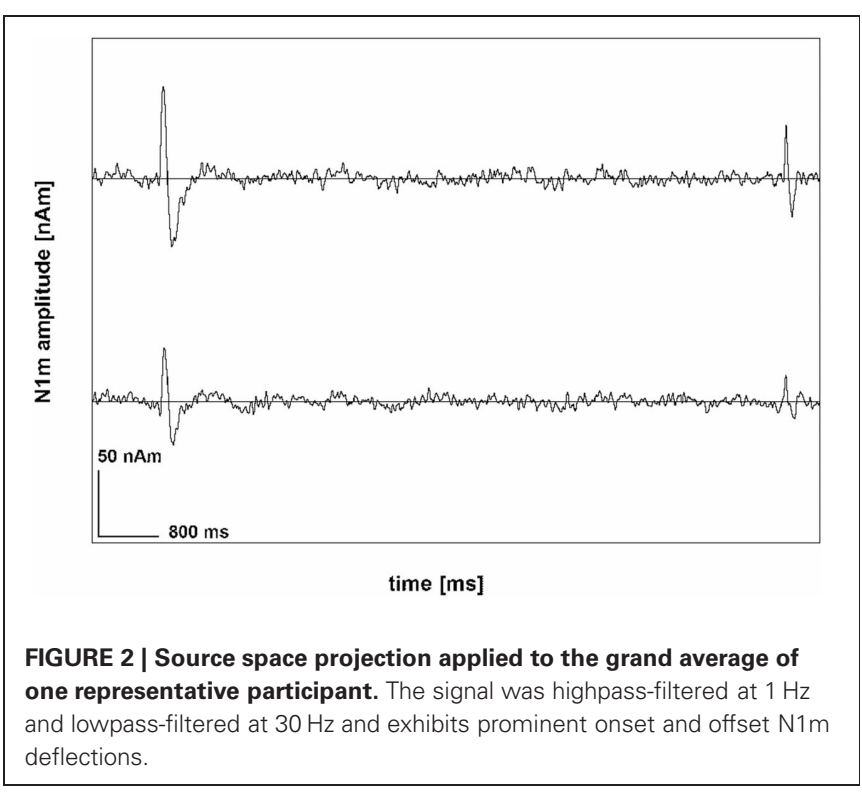


composite stimuli of the composite stimulus condition, as conditions for epochs of $500 \mathrm{~ms}$ duration. The modulation rate conditions were pooled in the averaging process. The epochs included a $200 \mathrm{~ms}$ pre-stimulus baseline interval. The N1m response peak was determined as the amplitude maximum within an analysis windows extending from $80 \mathrm{~ms}$ to $140 \mathrm{~ms}$ re-stimulus onset.

The N1m reciprocal inhibition ratio was defined as the ratio of source amplitude in the composite stimulus condition and the linear superposition sum of the source amplitudes in the three single stimulus conditions. Thus, larger reciprocal inhibition ratios indicate smaller amounts of reciprocal inhibition. The single stimulus condition N1m source amplitude data were submitted to a multivariate analysis of variance (SPSS GLM) with source amplitude as the dependent variable, hemisphere and tonal frequency as repeated measurement factors, and tinnitus status and musicality as grouping factors. Analysis of the data from the composite stimulus condition and of the reciprocal inhibition ratios was done accordingly, with hemisphere as a repeated measurement factor and tinnitus status and musicality as grouping factors.

\section{RESULTS}

In the single stimulus condition, there were two statistically significant main effects of N1m source amplitude, hemisphere $\left[T_{(1,31)}^{2}=0.879, p<0.0005\right]$ and tonal frequency $\left[T_{(2,30)}^{2}=\right.$ $1.021, p<0.0005]$. The mean $\mathrm{N} 1 \mathrm{~m}$ source amplitude, across tonal frequencies, was larger in the contralateral $(42.0 \mathrm{nAm})$ than in the ipsilateral hemisphere (30.5 nAm). Averaged across hemispheres, N1m amplitude was largest in the sub-edge condition (42.3 nAm), intermediate in the edge condition (35.0 nAm), and smallest in the (surrogate) tinnitus condition $(31.4 \mathrm{nAm})$. The same pattern was shown by tinnitus patients and healthy controls both in the contra- and the ipsilateral hemisphere (Figure 3A). The difference between tinnitus patients and controls did not attain significance and neither did the grouping factor of musicality or any of the interactions. When the analysis was rerun with age and low and high frequency hearing loss in the stimulated and the non-stimulated ear as covariates, the effect of frequency was preserved $\left[T_{(2,23)}^{2}=0.45, p<0.02\right]$, but the effect of hemisphere was not $\left[T_{(1,24)}^{2}=0.15\right.$, n.s. $]$. However, the interaction of musicality and hemisphere attained significance $\left[T_{(1,24)}^{2}=0.19, p<0.05\right]$. The difference between contraand ipsilateral hemisphere was larger for musicians than for non-musicians.

On the face of it and in accordance with Colding-Jorgensen et al. (1992); Jacobson et al. (1991); Jacobson and McCaslin (2003); and Walpurger et al. (2003); mean N1m source amplitude was smaller for patients than controls (Figure 3A), but the difference failed to attain significance, possibly because of the use of individually adjusted tonal stimulus frequencies which may have generated additional between-subject variance. After two outliers with exceptionally large N1m amplitudes were removed from the group of musicians without tinnitus and the analysis was rerun using a hierarchical sum-of-squares decomposition approach (SPSS GLM SSTYPES $=1$ ) which adjusted every term of the ANOVA model for the log of the individual audiometric edge frequency, not only the main effects of hemisphere $\left[T_{(1,25)}^{2}=\right.$
1.18, $p<0.0005]$ and frequency $\left[T_{(2,24)}^{2}=1.16, p<0.0005\right]$, but also the interactions between tinnitus status and musicality $\left[F_{(1,25)}=7.69, p<0.01\right]$, tinnitus status and hemisphere $\left[T_{(1,25)}^{2}=0.21, p<0.05\right]$, and tinnitus status, hemisphere, and frequency $\left[T_{(2,24)}^{2}=0.31, p<0.05\right]$ attained significance. The difference between patients and controls was larger for nonmusicians than musicians and larger for the ipsilateral than for the contralateral hemisphere, especially in the sub-edge condition. Simple effects testing revealed that patients showed marginally smaller N1m amplitudes than controls among nonmusicians $\left[F_{(1,25)}=3.80, p<0.07\right]$, for the ipsilateral hemisphere $\left[F_{(1,25)}=4.95, p<0.05\right]$, and for the sub-edge condition within the ipsilateral hemisphere $\left[F_{(1,25)}=9.73, p<0.005\right]$.

In the composite stimulus condition, the effects of hemisphere and musicality were statistically significant. N1m source amplitude was larger in the contralateral than in the ipsilateral hemisphere $\left[T_{(1,31)}^{2}=1.159, p<0.0005\right]$ and larger in musicians than in non-musicians $\left[F_{(1,31)}=4.578, p<0.05\right]$. The musicality-by-hemisphere interaction was marginally significant $\left[T_{(1,31)}^{2}=0.133, p<0.06\right]$. The hemisphere difference was marginally larger for musicians $(\mathrm{cH}: 88.0 \mathrm{nAm}, \mathrm{iH}: 59.8 \mathrm{nAm})$ than non-musicians (cH: $55.1 \mathrm{nAm}, \mathrm{iH}: 40.5 \mathrm{nAm}$ ).

Tinnitus patients and healthy controls differed with regard to N1m reciprocal inhibition. The N1m reciprocal inhibition score, i.e., the ratio of source amplitude in the composite stimulus condition and the superposition sum of the source amplitudes in the three single stimulus conditions, was significantly larger in patients than in healthy controls $\left[F_{(1,31)}=8.9, p<0.01\right]$. Thus, the patients showed less reciprocal inhibition than the controls (Figure 3B). The factors of musicality and hemisphere and all the possible interactions they were part of did not attain significance.

For comparison, Figure 3C shows single tone ASSR amplitudes and composite tone ASSR component amplitudes (Diesch et al., 2010a). In patients, ASSR amplitude was significantly larger in the tinnitus than in the edge condition $\left[F_{(1,34)}=5.7, p<\right.$ 0.025]. In controls, ASSR amplitude was significantly smaller in the edge $\left[F_{(1,34)}=20.2, p<0.005\right]$ and the surrogate tinnitus condition $\left[F_{(1,34)}=4.8, p<0.05\right]$ than in the sub-edge condition. There were significant interactions of mode of presentation (single, composite) and tinnitus status $\left[F_{(1,32)}=10.5, p<\right.$ 0.005 ] and mode of presentation, carrier frequency, and tinnitus status $\left[T_{(2,31)}^{2}=0.35, p<0.01\right]$. Inspection of the interactions shows that, except for the edge condition, ASSR amplitude was larger in the composite than in the single presentation condition in patients. Throughout, ASSR amplitude was smaller for the composite than the single condition in controls. The composite condition amplitude reduction in the edge condition was larger for controls than for patients.

\section{DISCUSSION}

When single tones were presented, the N1m source amplitude was larger in the hemisphere contralatersal than in the hemisphere ipsilateral to the stimulated ear. This laterality effect is in agreement with previous N1m findings (Pantev et al., 1998b). N1m amplitude was largest for sub-edge tonal frequencies, intermediate for edge frequencies, and smallest for (surrogate) tinnitus 


\section{A}

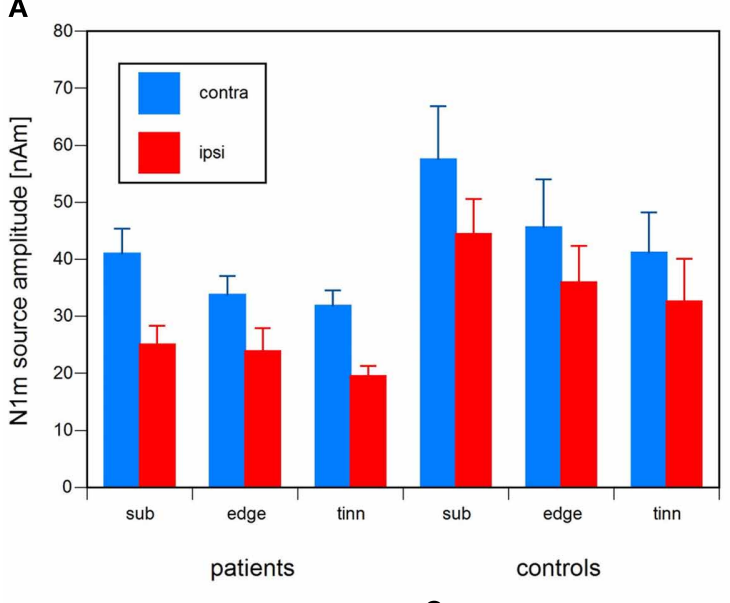

B

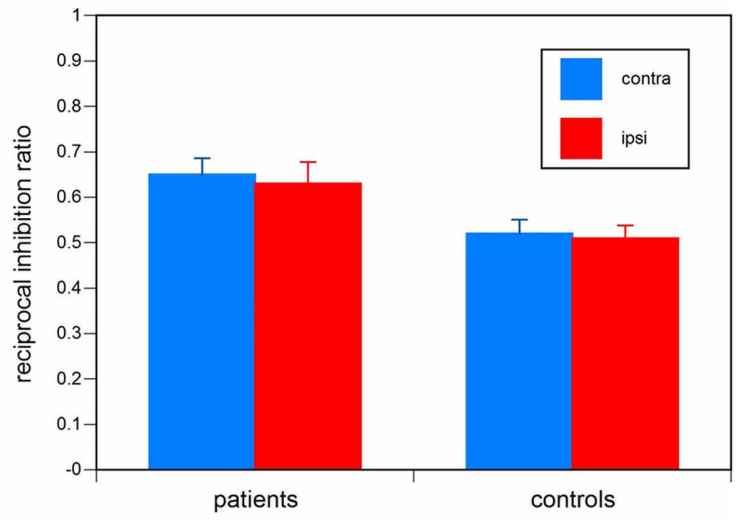

C

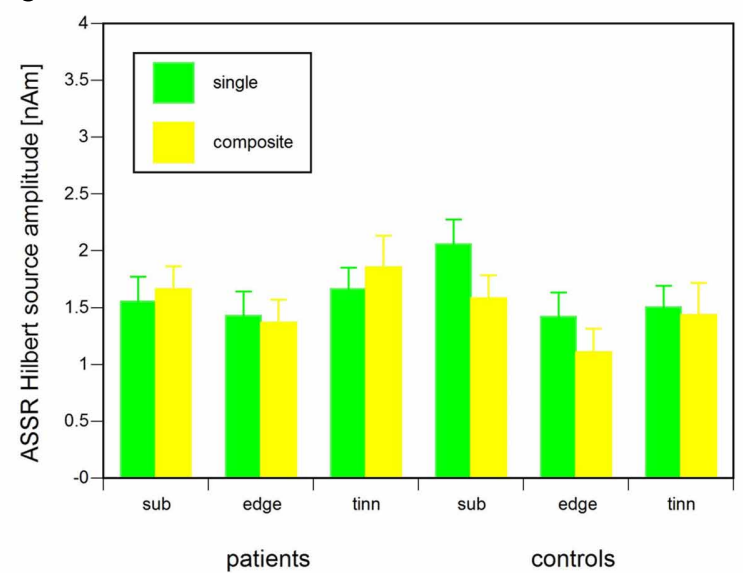

FIGURE 3 | (A) N1m source amplitude in the single stimulus condition for the contra- and the ipsilateral hemisphere, relative to the stimulated ear, in tinnitus patients and healthy controls for the tonal frequency conditions of sub-edge frequency, edge frequency, and (surrogate) tinnitus frequency. The error bars represent the standard error of measurement. (B) The reciprocal inhibition ratio, i.e., the ratio of source amplitude in the composite

stimulus condition and the linear superposition sum of the source amplitudes in the three single stimulus conditions, in the contra- and the ipsilateral hemisphere, relative to the stimulated ear, of tinnitus patients, and healthy controls. The error bars represent the standard error of measurement. (C) Sub-edge, edge, and (surrogate) tinnitus single and composite stimulus condition ASSR source amplitude in tinnitus patients and healthy controls [as reported in Diesch et al. (2010a)]. The error bars represent the standard error of measurement. frequencies. This is in agreement with the observation that, above approximately $1000 \mathrm{~Hz}, \mathrm{~N} 1 \mathrm{~m}$ amplitude decreases as a function of frequency (Pantev et al., 1995; Fujioka et al., 2002; Gabriel et al., 2004). These findings were preserved when age and hearing loss were entered as covariates into the analysis. N1m source amplitude was not larger in tinnitus patients than in healthy controls in any of the tonal frequency conditions, the (surrogate) tinnitus frequency included. If anything, N1m source amplitude was smaller for patients than controls, but this apparent difference, which is in agreement with previous reports on the amplitude of the N1 in tinnitus (Jacobson et al., 1991; Colding-Jorgensen et al., 1992; Jacobson and McCaslin, 2003; Walpurger et al., 2003), did not attain statistical significance as a main effect. This may be due to the additional between-subject variance generated by the individual adjustment of stimulus carrier frequencies. Simple effects testing after inclusion of the audiometric edge frequency into the ANOVA model showed that, among non-musicians, for the hemisphere ipsilateral to the stimulated ear, and for the sub-edge frequency within the ipsilateral hemisphere, patients exhibited smaller N1m amplitudes than controls. This partially accords with the findings of Colding-Jorgensen et al. (1992); Jacobson et al. (1991); Jacobson and McCaslin (2003); and Walpurger et al. (2003); and it is tempting to interpret it in terms of a refractory state that the tinnitus signal may produce for the N1m generator according to Jacobson and McCaslin (2003). However, the difference between patients and controls was largest in the sub-edge condition within the ipsilateral hemisphere, not the (surrogate) tinnitus condition. It could also be the result of the fact that stimulus level had to be lowered below $50 \mathrm{~dB}$ SL more often for patients than for controls.

As in the single stimulus condition, in the composite stimulus condition the N1m amplitude was larger in the hemisphere contralateral than in the hemisphere ipsilateral to the stimulated ear. While this difference was not significant in the single 
stimulus condition, N1m amplitude was larger in musicians than in non-musicians in the composite stimulus condition. This finding corresponds to previous reports concerning the processing of spectrally complex stimuli in musicians (Pantev et al., 1998a; Kuriki et al., 2006; Baumann et al., 2008; but see Schneider et al., 2002 and Lütkenhöner et al., 2006 for evidence to the contrary).

Qualitatively, the pattern of N1m source amplitude findings in patients and controls matches the one for ASSR source amplitude in controls obtained in earlier studies. In Diesch et al. (2010a), ASSR source amplitude in controls was smaller in the edge and the surrogate tinnitus frequency conditions than in the sub-edge condition and the edge and the surrogate tinnitus frequency condition did not differ significantly. However, in tinnitus patients, ASSR source amplitude was significantly larger in the tinnitus condition than in the edge condition. ASSR amplitude is affected by attention (Ross et al., 2004; Bidet-Caulet et al., 2007; Gander et al., 2007, 2010; Müller et al., 2009) and the perception of tinnitus and activity enhancement effects associated with tinnitus have frequently been interpreted in terms of attentional processes (Newman et al., 1997; Cuny et al., 2004; Zenner et al., 2006; Searchfield et al., 2007; Knobel and Sanchez, 2008; Gu et al., 2010; Rauschecker et al., 2010). Accordingly, it seems important to come to a conclusion on the the ASSR enhancement effect in tinnitus. Given that N1m attention enhancement effects are significantly larger than ASSR attention enhancement effects in healthy controls (Okamoto et al., 2011), if the ASSR amplitude enhancement effect in tinnitus were an effect of attention, an amplitude enhancement effects should also be found for the N1m. However, in the current study, the amplitude enhancement effect was conspicuously absent from the N1m source amplitude data. This renders it unlikely that the enhancement effect of tinnitus on ASSR amplitude is due to top-down effect of attention and strengthens the hypothesis of autoregulatory gain control being inherent to the functioning of the subcortical nuclei of the afferent auditory pathway and the primary and non-primary auditory cortex fields.

The finding that the N1m reciprocal inhibition score was significantly larger in patients than in healthy controls indicates that reciprocal inhibition was reduced in patients and structurally matches earlier ASSR findings. Diesch et al. (2010a) studied the ASSR to single AM tones and the ASSR to superpositions of three AM-tones differing in carrier and modulation frequency. Modulation frequency-specific ASSR components were recovered by bandpass filtering. Compared to the response to single AM-tones, ASSR components in the composite stimulus condition were reduced in amplitude in healthy controls, but increased in tinnitus patients. In controls,

\section{REFERENCES}

Andersson, G., Eriksson, J., Lundh, L. G., and Lyttkens, L. (2000). Tinnitus and cognitive interference: a stroop paradigm study. J. Speech Lang. Hear. Res. 43, 1168-1173.

Arthur, D. L., Lewis, P. S., Medvick, P. A., and Flynn, E. R. (1991). A neuromagnetic study of selective auditory attention. Electroencephalogr. Clin. Neurophysiol. 78, 348-360.

Baumann, S., Meyer, M., and Jancke, L. (2008). Enhancement of auditoryevoked potentials in musicians reflects an influence of expertise but not selective attention. J. Cogn. Neurosci. 20, 2238-2249.

multiple response components seemed to reciprocally inhibit one another, but in tinnitus patients there seemed to be reciprocal facilitation.

It may be argued that the reduction of reciprocal inhibition in tinnitus provides further evidence against the attention hypothesis. Attention affects sensory processing not only by increasing the gain, but also by increasing the selectivity of single unit receptive fields (Fritz et al., 2007) and of the response of populations of single units (Okamoto et al., 2007; Kauramäki et al., 2007; Neelon et al., 2011). As reciprocal inhibition among parallel sensory input streams is capable of sharpening the distinctiveness of each of them, the top-down control of reciprocal inhibition may be one of the mechanisms of attention. The reduction of reciprocal inhibition shown by tinnitus patients both with respect to the ASSR and the N1m is the opposite of what would be expected under the hypothesis of attention-directed modulation of sensory input streams.

The conclusion that attention does not account for the ASSR enhancement effect in tinnitus does not mean that there are no effects of tinnitus on attention and cognitive performance and no role of attention in tinnitus development and maintenance. Stroop paradigm, auditory working memory, and divided attention studies have provided evidence for attention and performance deficits in tinnitus patients (Andersson et al., 2000; Rossiter et al., 2006; Stevens et al., 2007) that suggests that tinnitus may curtail the focussing of attention on external stimuli. Neither does it mean that attention could not be involved in the plastic changes that generate gain change and activity enhancement effects in the primary afferent auditory pathway and the auditory cortex. The patients investigated in the present study on N1m amplitude and in the Diesch et al. (2010a) study on ASSR amplitude represented a chronic condition, with a reported mean duration of the tinnitus since its onset of 13 years, six months. While attention does not seem to account for tinnitus-related ASSR amplitude enhancement in this population, it is possible that this may be an altogether different matter in a population of acute patients. At least some forms of training-induced cortical plasticity (Polley et al., 2006; Fahle, 2009) may require top-down attentional control. This may also be true of the neuroplasticity that is induced by cochlear lesions and may result in tinnitus (Zenner et al., 2006).

\section{ACKNOWLEDGMENTS}

This research was supported by a grant of the Deutsche Forschungsgemeinschaft to Eugen Diesch and Andre Rupp (Di497/3-2). We would like to thank the reviewers of an earlier version of this paper for their insightful and constructive comments.

Bidet-Caulet, A., Fischer, C., Besle, J., Aguera, P. E., Giard, M. H., and Bertrand, O. (2007). Effects of selective attention on the electrophysiological representation of concurrent sounds in the human auditory cortex. J. Neurosci. 27, 9252-9261.

Colding-Jorgensen, E., Lauritzen, M., Johnsen, N. J., Mikkelsen, K. B., and Saermark, K. (1992). On the evidence of auditory evoked magnetic fields as an objective measure of tinnitus. Electroencephalogr. Clin. Neurophysiol. 83, 322-327.

Cuny, C., Norena, A., El Massioui, F., and Chery-Croze, S. (2004). Reduced attention shift in response to auditory changes in subjects 
with tinnitus. Audiol. Neurootol. 9, 294-302.

Dau, T., Verhey, J., and Kohlrausch, A. (1999). Intrinsic envelope fluctuations and modulation-detection thresholds for narrow-band noise carriers. J. Acoust. Soc. Am. 106, 2752-2760.

Diesch, E., Andermann, M., Flor, H., and Rupp, A. (2010a). Interaction among the components of multiple auditory steady-state responses: enhancement in tinnitus patients, inhibition in controls. Neuroscience 167, 540-553.

Diesch, E., Andermann, M., Flor, H., and Rupp, A. (2010b). Functional and structural aspects of tinnitusrelated enhancement and suppression of auditory cortex activity. Neuroimage 50, 1545-1559.

Diesch, E., and Luce, T. (1997). Magnetic fields elicited by tones and vowel formants reveal tonotopy and nonlinear summation of cortical activation. Psychophysiology 34, 501-510.

Diesch, E., Struve, M., Rupp, A., Ritter, S., Hulse, M., and Flor, H. (2004). Enhancement of steady-state auditory evoked magnetic fields in tinnitus. Eur. J. Neurosci. 19, 1093-1104.

Eggermont, J. J., and Roberts, L. E. (2004). The neuroscience of tinnitus. Trends Neurosci. 27, 676-682.

Fahle, M. (2009). Perceptual learning and sensomotor flexibility: cortical plasticity under attentional control? Philos. Trans. R. Soc. Lond. B Biol. Sci. 364, 313-319.

Fritz, J. B., Elhilali, M., David, S. V., and Shamma, S. A. (2007). Auditory attention-focusing the searchlight on sound. Curr. Opin. Neurobiol. 17, 437-455.

Fujiki, N., Jousmaki, V., and Hari, R. (2002). Neuromagnetic responses to frequency-tagged sounds: a new method to follow inputs from each ear to the human auditory cortex during binaural hearing. J. Neurosci. 22, RC205.

Fujioka, T., Kakigi, R., Gunji, A., and Takeshima, Y. (2002). The auditory evoked magnetic fields to very high frequency tones. Neuroscience 112, 367-381.

Fujiwara, N., Nagamine, T., Imai, M., Tanaka, T., and Shibasaki, H. (1998). Role of the primary auditory cortex in auditory selective attention studied by whole-head neuromagnetometer. Brain Res. Cogn. Brain Res. 7, 99-109.

Gabriel, D., Veuillet, E., Ragot, R., Schwartz, D., Ducorps, A., Norena, A., Durrant, J. D., Bonmartin, A., Cotton, F., and Collet, L. (2004).
Effect of stimulus frequency and stimulation site on the N1m response of the human auditory cortex. Hear. Res. 197, 55-64.

Gander, P. E., Bosnyak, D. J., and Roberts, L. E. (2010). Evidence for modality-specific but not frequency-specific modulation of human primary auditory cortex by attention. Hear. Res. 268, 213-226.

Gander, P. E., Bosnyak, D. J., Wolek, R., and Roberts, L. E. (2007). Modulation of the $40-\mathrm{Hz}$ auditory steady-state response by attention during acoustic training. Int. Congr. Ser. 1300, 37-40.

Godey, B., Schwartz, D., de Graaf, J. B., Chauvel, P., and LiegeoisChauvel, C. (2001). Neuromagnetic source localization of auditory evoked fields and intracerebral evoked potentials: a comparison of data in the same patients. Clin. Neurophysiol. 112, 1850-1859.

Goebel, G., and Hiller, W. (1994). The tinnitus questionnaire. A standard instrument for grading the degree of tinnitus. Results of a multicenter study with the tinnitus questionnaire. HNO 42, 166-172.

Goebel, G., and Hiller, W. (1998). Der Tinnitus-Fragebogen. Göttingen: Hogrefe.

Gordon, E. (1989). Advanced Measures of Music Audiation. Chicago, IL: GIA Publications.

Gordon, E. (1998). Introduction to Research and the Psychology of Music. Chicago, IL: GIA Publications.

Gu, J. W., Halpin, C. F., Nam, E. C., Levine, R. A., and Melcher, J. R. (2010). Tinnitus, diminished sound-level tolerance, and elevated auditory activity in humans with clinically normal hearing sensitivity. J. Neurophysiol. 104, 3361-3370.

Gutschalk, A., Mase, R., Roth, R., Ille, N., Rupp, A., Hähnel, S., Picton, T. W., and Scherg, M. (1999). Deconvolution of $40 \mathrm{~Hz}$ steadystate fields reveals two overlapping source activities of the human auditory cortex. Clin. Neurophysiol. 110, 856-868.

Hämälainen, M., Hari, R., Ilmoniemi, R., Knuutila, J., and Lounasmaa, O. V. (1993). Magnetoencephalography - theory, instrumentation, and applications to noninvasive studies of the working human brain. Rev. Mod. Phys. 65, 413-497.

Hallam, R. S. (1996). Manual of the Tinnitus Questionnaire (TQ). London, UK: Psychological Corporation.

Hallam, R. S., Jakes, S. C., and Hinchcliffe, R. (1988). Cognitive variables in tinnitus annoyance.
Br. J. Clin. Psychol. 27(Pt 3), 213-222.

Henry, J. A., Fausti, S. A., Flick, C. L., Helt, W. J., and Ellingson, R. M. (2000). Computer-automated clinical technique for tinnitus quantification. Am. J. Audiol. 9, 36-49.

Henry, J. A., and Meikle, M. B. (2000). Psychoacoustic measures of tinnitus. J. Am. Acad. Audiol. 11, 138-155.

Hillyard, S. A., Hink, R. F., Schwent, V. L., and Picton, T. W. (1973) Electrical signs of selective attention in the human brain. Science 182 , 177-180.

Hillyard, S. A., Vogel, E. K., and Luck, S. J. (1998). Sensory gain control (amplification) as a mechanism of selective attention: electrophysiological and neuroimaging evidence [In Process Citation]. Philos. Trans. R. Soc. Lond. B Biol. Sci. 353, 1257-1270.

Jacobson, G. P., Ahmad, B. K., Moran, J., Newman, C. W., Tepley, N., and Wharton, J. (1991). Auditory evoked cortical magnetic field (M100-M200) measurements in tinnitus and normal groups. Hear. Res. 56, 44-52.

Jacobson, G. P., and McCaslin, D. L. (2003). A reexamination of the long latency N1 response in patients with tinnitus. J. Am. Acad. Audiol. 14, 393-400.

Jen, P. H., Chen, Q. C., and Wu, F. J. (2002). Interaction between excitation and inhibition affects frequency tuning curve, response size and latency of neurons in the auditory cortex of the big brown bat, Eptesicus fuscus. Hear. Res. 174, 281-289.

John, M. S., Lins, O. G., Boucher, B. L. and Picton, T. W. (1998). Multiple auditory steady-state responses (MASTER): stimulus and recording parameters. Audiology 37, 59-82.

Kadner, A., Viirre, E., Wester, D. C., Walsh, S. F., Hestenes, J., Vankov, A., and Pineda, J. A. (2002). Lateral inhibition in the auditory cortex: an EEG index of tinnitus? Neuroreport 13, 443-446.

Kaneko, K., Fujiki, N., and Hari, R. (2003). Binaural interaction in the human auditory cortex revealed by neuromagnetic frequency tagging: no effect of stimulus intensity. Hear. Res. 183, 1-6

Kaur, S., Lazar, R., and Metherate, R. (2004). Intracortical pathways determine breadth of subthreshold frequency receptive fields in primary auditory cortex. $J$. Neurophysiol. 91, 2551-2567.
Kauramäki, J., Jaaskelainen, I. P., and Sams, M. (2007). Selective attention increases both gain and feature selectivity of the human auditory cortex. PLoS ONE 2:e909. doi: 10.1371/journal.pone.0000909

Knobel, K. A., and Sanchez, T. G. (2008). Influence of silence and attention on tinnitus perception. Otolaryngol. Head Neck Surg. 138, 18-22.

Kohlrausch, A., Fassel, R., van der Heijden, M., Kortekaas, R., van de Par, S., Oxenham, A., and Püschel, D. (1997). Detection of tones in lownoise noise: further evidence for the role of envelope fluctuations. Acustica Acta Acustica 83, 659-669.

Kopp-Scheinpflug, C., Dehmel, S., Dorrscheidt, G. J., and Rubsamen, R. (2002). Interaction of excitation and inhibition in anteroventral cochlear nucleus neurons that receive large endbulb synaptic endings. J. Neurosci. 22, 11004-11018.

Kuriki, S., Kanda, S., and Hirata, Y. (2006). Effects of musical experience on different components of MEG responses elicited by sequential piano-tones and chords. J. Neurosci. 26, 4046-4053.

Lins, O. G., and Picton, T. W. (1995). Auditory steady-state responses to multiple simultaneous stimuli. Electroencephalogr. Clin. Neurophysiol. 96, 420-432.

Lins, O. G., Picton, T. W., Boucher, B. L., Durieux-Smith, A., Champagne, S. C., Moran, L. M., Perez-Abalo, M. C., Martin, V., and Savio, G. (1996). Frequency-specific audiometry using steady-state responses. Ear Hear. 17, 81-96.

Lütkenhöner, B., Seither-Preisler, A., and Seither, S. (2006). Piano tones evoke stronger magnetic fields than pure tones or noise, both in musicians and non-musicians. Neuroimage 30, 927-937.

Lütkenhöner, B., and Steinsträter, O. (1998). High-precision neuromagnetic study of the functional organization of the human auditory cortex. Audiol. Neurootol. 3, 191-213.

Mäkelä, J. P., and Hari, R. (1987). Evidence for cortical origin of the $40 \mathrm{~Hz}$ auditory evoked response in man. Electroencephalogr. Clin. Neurophysiol. 66, 539-546.

Metherate, R., Kaur, S., Kawai, H., Lazar, R., Liang, K., and Rose, H. J. (2005). Spectral integration in auditory cortex: mechanisms and modulation. Hear. Res. 206, 146-158.

Müller, C. M., and Scheich, H. (1988). Contribution of GABAergic inhibition to the response characteristics of auditory units in the 
avian forebrain. J. Neurophysiol. 59, 1673-1689.

Müller, N., Schlee, W., Hartmann, T., Lorenz, I., and Weisz, N. (2009). Top-down modulation of the auditory steady-state response in a task-switch paradigm. Front. Hum. Neurosci. 3:1. doi: 10.3389/neuro.09.001.2009

Näätänen, R. (1992). Attention and Brain Function. Hillsdale, NJ: Erlbaum.

Näätänen, R., and Picton, T. (1987). The N1 wave of the human electric and magnetic response to sound: a review and an analysis of the component structure. Psychophysiology $24,375-425$.

Neelon, M. F., Williams, J., and Garell, P. C. (2011). Elastic attention: enhanced, then sharpened response to auditory input as attentional load increases. Front. Hum. Neurosci. 5:41. doi: 10.3389/fnhum.2011.00041

Newman, C. W., Wharton, J. A., and Jacobson, G. P. (1997). Self-focused and somatic attention in patients with tinnitus. J. Am. Acad. Audiol. 8, 143-149.

Norena, A., Cransac, H., and CheryCroze, S. (1999). Towards an objectification by classification of tinnitus. Clin. Neurophysiol. 110, 666-675.

Norena, A. J. (2010). An integrative model of tinnitus based on a central gain controlling neural sensitivity. Neurosci. Biobehav. Rev. 35, 1089-1109.

Okamoto, H., Stracke, H., Bermudez, P., and Pantev, C. (2011). Sound processing hierarchy within human auditory cortex. J. Cogn. Neurosci. $23,1855-1863$.

Okamoto, H., Stracke, H., Wolters, C. H., Schmael, F., and Pantev, C. (2007). Attention improves population-level frequency tuning in human auditory cortex. $J$. Neurosci. 27, 10383-10390.

Pantev, C., Bertrand, O., Eulitz, C., Verkindt, C., Hampson, S., Schuierer, G., and Elbert, T. (1995). Specific tonotopic organizations of different areas of the human auditory cortex revealed by simultaneous magnetic and electric recordings. Electroencephalogr. Clin. Neurophysiol. 94, 26-40.

Pantev, C., Oostenveld, R., Engelien, A., Ross, B., Roberts, L. E., and Hoke, M. (1998a). Increased auditory cortical representation in musicians. Nature 392, 811-814.

Pantev, C., Ross, B., Berg, P., Elbert, T., and Rockstroh, B. (1998b). Study of the human auditory cortices using a whole-head magnetometer: left vs. right hemisphere and ipsilateral vs. contralateral stimulation. Audiol. Neurootol. 3, 183-190.

Parra, L. C., and Pearlmutter, B. A. (2007). Illusory percepts from auditory adaptation. J. Acoust. Soc. Am. 121, 1632-1641.

Picton, T. W., and Hillyard, S. A. (1974). Human auditory evoked potentials. II. Effects of attention. Electroencephalogr. Clin. Neurophysiol. 36, 191-199.

Polley, D. B., Steinberg, E. E., and Merzenich, M. M. (2006). Perceptual learning directs auditory cortical map reorganization through top-down influences. $J$. Neurosci. 26, 4970-4982.

Rauschecker, J. P., Leaver, A. M., and Muhlau, M. (2010). Tuning out the noise: limbic-auditory interactions in tinnitus. Neuron 66, 819-826.

Rhode, W. S., and Greenberg, S. (1994). Encoding of amplitude modulation in the cochlear nucleus of the cat. $J$. Neurophysiol. 71, 1797-1825.

Rif, J., Hari, R., Hamalainen, M. S., and Sams, M. (1991). Auditory attention affects two different areas in the human supratemporal cortex. Electroencephalogr. Clin. Neurophysiol. 79, 464-472.

Robinson, S. E. (1989). "Theory and properties of lead field synthesis analysis," in Advances in Biomagnetism, eds S. J. Williamson, M. Hoke, G. Stroink, and M. Kotani (New York, NY: Plenum Press), 599-602.

Ross, B., Borgmann, C., Draganova, R., Roberts, L. E., and Pantev, C. (2000). A high-precision magnetoencephalographic study of human auditory steady-state responses to amplitude-modulated tones. $J$. Acoust. Soc. Am. 108, 679-691.

Ross, B., Picton, T. W., Herdman, A. T., and Pantev, C. (2004). The effect of attention on the auditory steady-state response. Neurol. Clin. Neurophysiol. 2004, 22.

Ross, B., Picton, T. W., and Pantev, C. (2002). Temporal integration in the human auditory cortex as represented by the development of the steady-state magnetic field. Hear. Res. 165, 68-84.

Rossiter, S., Stevens, C., and Walker, G. (2006). Tinnitus and its effect on working memory and attention. $J$. Speech Lang. Hear. Res. 49, 150-160.

Salvi, R. J., Wang, J., and Ding, D. (2000). Auditory plasticity and hyperactivity following cochlear damage. Hear. Res. 147, 261-274.
Shamma, S. A., and Symmes, D. (1985). Patterns of inhibition in auditory cortical cells in awake squirrel monkeys. Hear. Res. 19, 1-13.

Schneider, P., Scherg, M., Dosch, H. G., Specht, H. J., Gutschalk, A., and Rupp, A. (2002). Morphology of Heschl's gyrus reflects enhanced activation in the auditory cortex of musicians. Nat. Neurosci. 5 , 688-694.

Schoonhoven, R., Boden, C. J., Verbunt, J. P., and de Munck, J. C. (2003). A whole head MEG study of the amplitude-modulation-following response: phase coherence, group delay and dipole source analysis. Clin. Neurophysiol. 114, 2096-2106.

Schulze, H., and Langner, G. (1999). Auditory cortical responses to amplitude modulations with spectra above frequency receptive fields: evidence for wide spectral integration. J. Comp. Physiol. A 185, 493-508.

Searchfield, G. D., Morrison-Low, J., and Wise, K. (2007). Object identification and attention training for treating tinnitus. Prog. Brain Res. $166,441-460$.

Steinschneider, M., Schroeder, C. E., Arezzo, J. C., and Vaughan, H. G. Jr. (1994). Speech-evoked activity in primary auditory cortex: effects of voice onset time. Electroencephalogr. Clin. Neurophysiol. 92, 30-43.

Stevens, C., Walker, G., Boyer, M., and Gallagher, M. (2007). Severe tinnitus and its effect on selective and divided attention. Int. J. Audiol. 46, 208-216.

Suga, N. (1995). Sharpening of frequency tuning by inhibition in the central auditory system: tribute to Yasuji Katsuki. Neurosci. Res. 21, 287-299.

Sun, W., Lu, J., Stolzberg, D., Gray, L., Deng, A., Lobarinas, E., and Salvi, R. J. (2009). Salicylate increases the gain of the central auditory system. Neuroscience 159, 325-334.

Sutter, M. L., Schreiner, C. E., McLean, M., O'connor, K. N., and Loftus, W. C. (1999). Organization of inhibitory frequency receptive fields in cat primary auditory cortex. $J$. Neurophysiol. 82, 2358-2371.

Syka, J. (2002). Plastic changes in the central auditory system after hearing loss, restoration of function, and during learning. Physiol. Rev. 82, 601-636.

Vater, M., Habbicht, H., Kossl, M., and Grothe, B. (1992). The functional role of GABA and glycine in monaural and binaural processing in the inferior colliculus of horseshoe bats. J. Comp. Physiol. A 171, 541-553.

Walpurger, V., Hebing-Lennartz, G., Denecke, H., and Pietrowsky, R. (2003). Habituation deficit in auditory event-related potentials in tinnitus complainers. Hear. Res. 181, 57-64.

Wienbruch, C., Paul, I., Weisz, N., Elbert, T., and Roberts, L. E. (2006). Frequency organization of the $40-\mathrm{Hz}$ auditory steadystate response in normal hearing and in tinnitus. Neuroimage 33, 180-194.

Winkowski, D. E., and Knudsen, E. I. (2006). Top-down gain control of the auditory space map by gaze control circuitry in the barn owl. Nature 439, 336-339.

Winkowski, D. E., and Knudsen, E. I. (2008). Distinct mechanisms for top-down control of neural gain and sensitivity in the owl optic tectum. Neuron 60, 698-708.

Woldorff, M. G., Gallen, C. C., Hampson, S. A., Hillyard, S. A., Pantev, C., Sobel, D., and Bloom, F. E. (1993). Modulation of early sensory processing in human auditory cortex during auditory selective attention. Proc. Natl. Acad. Sci. U.S.A. 90, 8722-8726.

Zenner, H. P., Pfister, M., and Birbaumer, N. (2006). Tinnitus sensitization: sensory and psychophysiological aspects of a new pathway of acquired centralization of chronic tinnitus. Otol. Neurotol. 27, 1054-1063.

Conflict of Interest Statement: The authors declare that the research was conducted in the absence of any commercial or financial relationships that could be construed as a potential conflict of interest.

Received: 26 December 2011; accepted: 03 May 2012; published online: 21 May 2012.

Citation: Diesch E, Andermann $M$ and Rupp A (2012) Is the effect of tinnitus on auditory steady-state response amplitude mediated by attention?. Front. Syst. Neurosci. 6:38. doi: 10.3389/fnsys. 2012.00038

Copyright (C) 2012 Diesch, Andermann and Rupp. This is an open-access article distributed under the terms of the Creative Commons Attribution Non Commercial License, which permits non-commercial use, distribution, and reproduction in other forums, provided the original authors and source are credited. 